\title{
Usefulness of selective renal artery embolization for urinary fistula following partial nephrectomy: Two case reports
}

\author{
TAKAHIRO NOHARA $^{1}$, SATOKO MATSUYAMA ${ }^{1}$, SHOHEI KAWAGUCHI ${ }^{1}$, \\ TOHRU MIYAGI $^{1}$, CHIKASHI SETO $^{1}$ and KENTARO MOCHIZUKI ${ }^{2}$ \\ Departments of ${ }^{1}$ Urology and ${ }^{2}$ Radiology, Toyama Prefectural Central Hospital, Toyama 930-8550, Japan
}

Received May 11, 2015; Accepted February 26, 2016

DOI: $10.3892 /$ mco.2016.885

\begin{abstract}
The present study reported two cases in which selective artery embolization were identified to assist in resolving urinary fistulae following partial nephrectomies. The first case involved a 51-year-old male who received a mini-incision partial nephrectomy with renorrhaphy. Following the operation, urine continued to discharge from the retroperitoneal drain. Selective renal artery embolization of the upper calyx at post-operation day 20 was highly effective and urine output from the drain stopped immediately. Case 2 involved a 66-year-old male, who also suffered from a urinary fistula following a partial nephrectomy. Selective renal artery embolization performed at post-operation day 21 was again effective. In each case, the upper calyx was separated from the renal pelvis. These cases demonstrated that suturing of the collecting system and renal parenchyma may result in the separation of the urine pathway, and that selective renal artery embolization appears to be a highly effective treatment in such cases.
\end{abstract}

\section{Introduction}

Partial nephrectomy (PN) is the most commonly used procedure for the treatment of clinical stage T1a renal cancer. Furthermore, evidence reveals that patients undergoing radical nephrectomy have a higher overall mortality and cardiovascular morbidity rate compared with those undergoing PN, even though their effectiveness in cancer control is equivalent $(1,2)$.

Correspondence to: Dr Takahiro Nohara, Department of Urology, Toyama Prefectural Central Hospital, 2-2-78 Nishi-nagae, Toyama 930-8550, Japan

E-mail: t_nohara704@yahoo.co.jp

Abbreviations=: PN, partial nephrectomy; POD, post-operation day; RCC, renal cell carcinoma; TAE, transarterial embolization; UF, urinary fistula

Key words: complication, partial nephrectomy, renal tumor, selective renal artery embolization, urinary fistula
However, PN results in urinary fistula (UF) in 1-6\% of patients $(3,4)$. The majority of these patients are forced to undergo lengthy hospital stays, since perinephric drains cannot be removed due to of persistent urine drainage. An effective management strategy for UF remains to be established.

The present study reported two cases in which selective artery embolization was useful for the treatment of UF following PN.

\section{Case reports}

Case 1. A 51-year-old male, diagnosed with renal cell carcinoma (RCC) in the right kidney (Fig. 1), received mini-incision PN with renorrhaphy at Toyama Prefectural Central Hospital (Toyama, Japan). Following the operation, urine discharge of $\sim 100 \mathrm{ml}$ per day continued from the retroperitoneal drain. Although ureteral stenting was performed at post-operation day (POD) 6, the quantity of drainage did not decrease. A computed tomography $(\mathrm{CT})$ at POD 13 revealed urine leakage in the upper part of the kidney. Furthermore, the upper calyx was completely separated from the middle/lower calyx and the renal pelvis. The upper calyx was not enlarged and it was considered unlikely that percutaneous drainage could be successfully performed. Since the UF could not be treated unless effective urine drainage was obtained, it was unavoidable to perform an alternative approach, including nephrectomy or transarterial embolization (TAE) of the entire kidney. However, the decision was made to perform selective TAE of the upper calyx at POD 20. Following this procedure, urine output from the drain stopped immediately. Although fever was observed for a few days, the patient was able to leave Toyama Prefectural Central Hospital at POD 29. The serum creatinine level was slightly increased (1.12 to $1.21 \mathrm{mg} / \mathrm{dl})$. Since discharge, no further complications or RCC recurrences have occurred for 3 years.

Case 2. A 66-year-old male, with a diagnosis of RCC in the left kidney (Fig. 2), received mini-incision PN with renorrhaphy at Toyama Prefectural Central Hospital. Following the operation, urine discharge of $\sim 50-100 \mathrm{ml}$ per day continued from the retroperitoneal drain. Although ureteral stenting was performed at POD 7, the quantity of drainage did not decrease. A CT at POD 12 revealed urine leakage in an upper part of the kidney. Furthermore, the upper calyx was completely separated from 


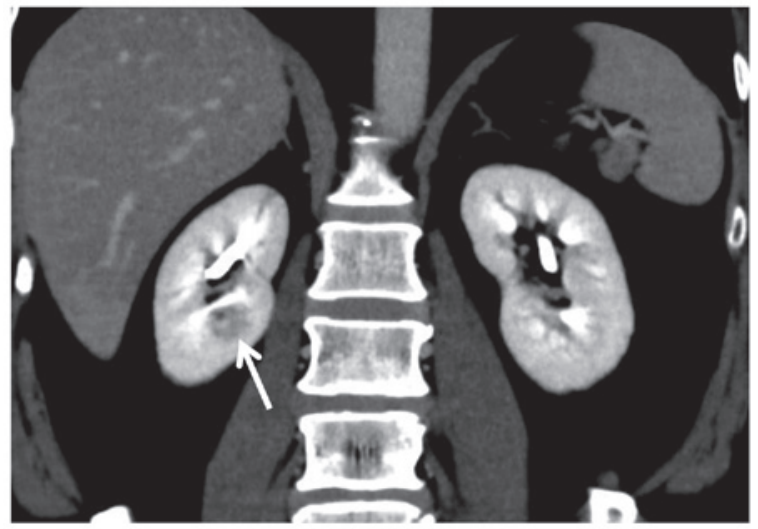

Figure 1. Pre-operative computed tomography of case 1. A small renal tumor is shown in the middle part of the right kidney (arrow).

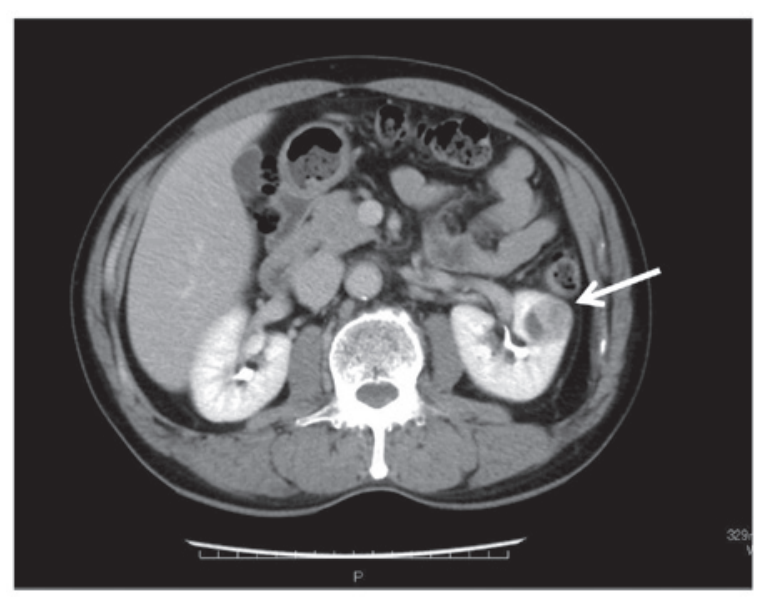

Figure 2. Pre-operative computed tomography of case 2. A small renal tumor is shown in the middle part of the right kidney (arrow).

the middle/lower calyx and the renal pelvis (Fig. 3), and it was slightly enlarged. This suggested that percutaneous drainage would be difficult to achieve, and therefore, we considered that selective TAE of the upper section of the kidney, as previously described in case 1, may be effective. This procedure was performed at POD 21 (Fig. 4) and resulted in the immediate cessation of urine output from the drain. Although fever was observed for a few days, the patient was able to leave Toyama Prefectural Central Hospital on POD 26. The serum creatinine level was slightly increased $(0.99$ to $1.06 \mathrm{mg} / \mathrm{dl})$. Since discharge, no complications and RCC recurrence have occurred for 2 years.

\section{Discussion}

Management of UF following PN can present serious difficulties. Textbooks state that most UF cases will resolve with several weeks of conservative therapy unless there is distal obstruction to the site of leakage (5), and medical literature demonstrates that many cases of UF are cured only by observation (3). Meeks et al (6) described 21 cases of UF, where a long mean duration of leakage of 53 days was noted. This previous study demonstrates that once UF occurs, patients are often confined to hospital for lengthy time periods.

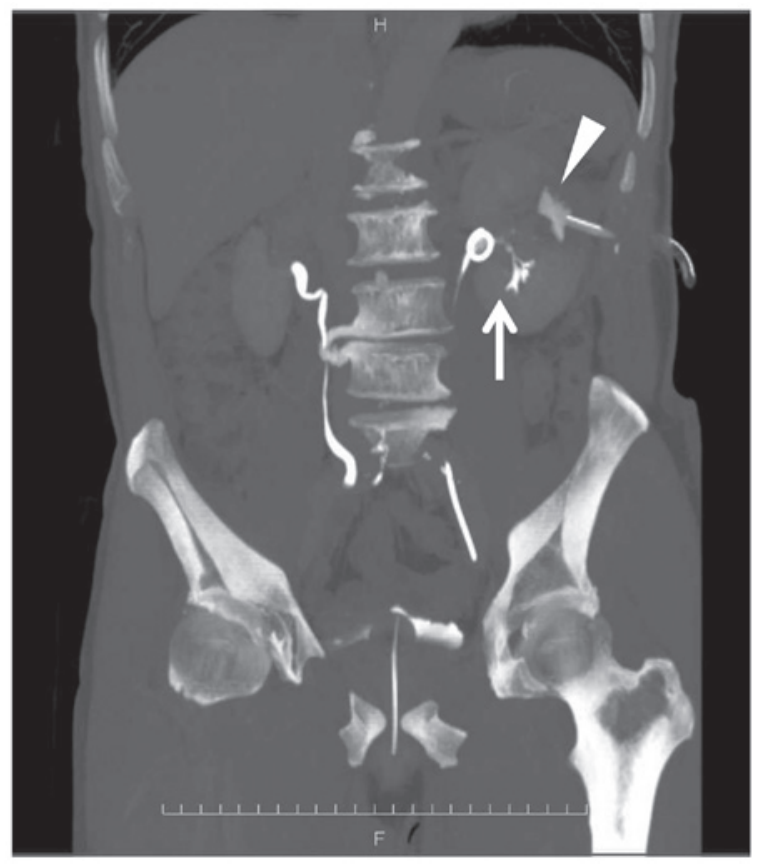

Figure 3. Post-operative computed tomography of case 2. A urinary fistula was recognized in the upper calyx of the kidney (triangle), which was completely separated from the middle/lower calyx and the renal pelvis (arrow).

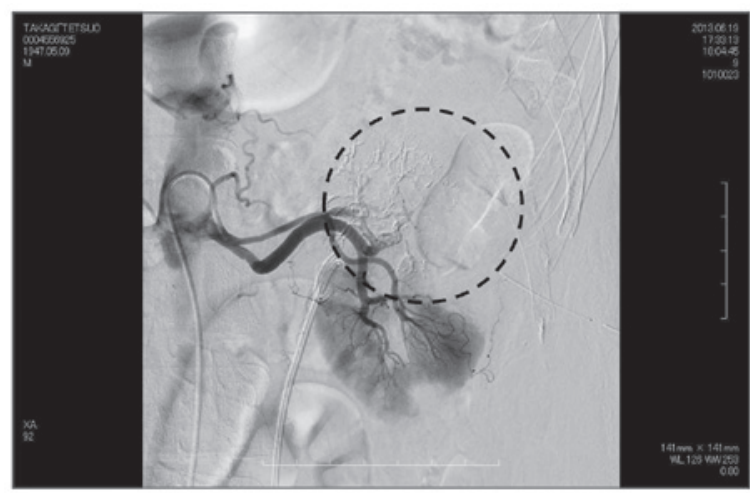

Figure 4. Renal angiography just after selective transarterial embolization of the apical branch of the left kidney. Vascular perfusion in the upper part of the kidney (the area in the black circle) was completely blocked.

In the two cases described in the present study, the upper calyx was completely separated from the middle/lower calyx, and therefore, prevented successful drainage of the upper calyx. It was suspected that deep suture of the renal parenchyma and/ or the collecting system during PN resulted in the upper calyx separating from the renal pelvis in each case. For the treatment of UF, nephrectomy or TAE of the entire kidney was considererd. However, selective TAE was attempted in order to resolve UF and to preserve renal function, and was successful in stopping urine discharge from the retroperitoneal drain.

To the best of our knowledge, only 4 cases of selective TAE in the treatment of UF have been previously reported $(7,8)$. In one of these cases, urinary drainage persisted from the remnant of the lower collecting system following hemi-nephrectomy of the lower-half of a duplex kidney (7). The other three cases involved horseshoe kidneys, which received half-side nephrectomies with incisions of the isthmus, and urinary drainage 
continued from the residual parenchyma that had originally drained into the renal pelvis of the resected side (8). In all four cases, no drainage pathways from the site of UF were observed and selective TAE was successful.

Therefore, it was observed that UF is unable to spontaneously resolve if good drainage pathways do not exist. Finally, from the two cases presented in the present study, the following was concluded: i) Suture of the collecting system and renal parenchyma in PN may result in the separation of the renal calyx from the renal pelvis; ii) Selective TAE of the renal parenchyma in UF sites appeared to be a highly effective treatment in such cases.

\section{References}

1. Thompson RH, Boorjian SA, Lohse CM, Leibovich BC, Kwon ED, Cheville JC and Blute ML: Radical nephrectomy for pT1a renal masses may be associated with decreased overall survival compared with partial nephrectomy. J Urol 179: 468-471; discussion 472-473, 2008.
2. Huang WC, Elkin EB, Levey AS, Jang TL and Russo P: Partial nephrectomy versus radical nephrectomy in patients with small renal tumors-is there a difference in mortality and cardiovascular outcomes? J Urol 181: 55-61; discussion 61-62, 2009.

3. Kundu SD, Thompson RH, Kallingal GJ, Cambareri G and Russo P: Urinary fistulae after partial nephrectomy. BJU Int 106: 1042-1044, 2010

4. Minervini A, Vittori G, Antonelli A, Celia A, Crivellaro S, Dente D, Di Santo V, Frea B, Gacci M, Gritti A, et al: Open versus robotic-assisted partial nephrectomy: A multicenter comparison study of perioperative results and complications. World J Urol 32: 287-293, 2014.

5. Kavoussi LR, Schwartz MJ and Gill IS: Laparoscopic surgery of the kidney. In: Kavoussi LR, Novick AC, Partin AW and Peters CA (eds). Campbell's Urology. 10th edition. Elsevier Saunders, Philadelphia, pp 1668, 2012.

6. Meeks JJ, Zhao LC, Navai N, Perry KT Jr, Nadler RB and Smith ND: Risk factors and management of urine leaks after partial nephrectomy. J Urol 180: 2375-2378, 2008.

7. Mitty HA, Dan SJ, Goldman HJ and Glickman SI: Urinary fistulas after partial nephrectomy: Treatment by segmental renal embolization. AJR Am J Roentgenol 141: 101-103, 1983.

8. Stefani P, Selli C, Nicita G, Lapini A and Dami A: Treatment of urinary fistulas after resection of horseshoe kidneys by selective arterial embolization. Cardiovasc Intervent Radiol 12: 18-21, 1989. 